\title{
A simple additive staging system for newly diagnosed multiple myeloma
}

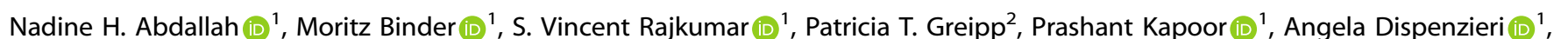

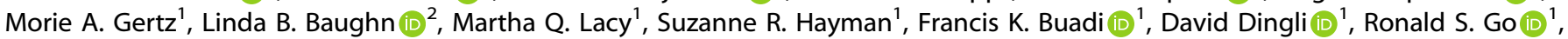
Yi L. Hwa', Amie L. Fonder ${ }^{1}$, Miriam A. Hobbs ${ }^{1}$, Yi Lin (D) ${ }^{1}$, Nelson Leung ${ }^{1,3}$, Taxiarchis Kourelis (D) ${ }^{1}$, Rahma Warsame ${ }^{1}$,

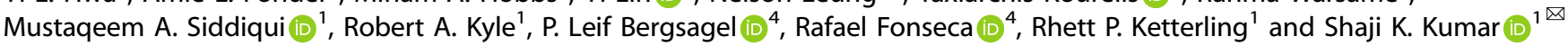

(c) The Author(s) 2022

Risk stratification in multiple myeloma is important for prognostication, patient selection for clinical trials, and comparison of treatment approaches. We developed and validated a staging system that incorporates additional FISH abnormalities not included in the R-ISS and reflects the additive effects of co-occurring high-risk disease features. We first evaluated the prognostic value of predefined cytogenetic and laboratory abnormalities in 2556 Mayo Clinic patients diagnosed between February 2004 and June 2019. We then used data from 1327 patients to develop a risk stratification model and validated this in 502 patients enrolled in the MMRF CoMMpass study. On multivariate analysis, high-risk IgH translocations [risk ratio (RR): 1.7], 1q gain/amplification (RR: 1.4), chromosome17 abnormalities (RR: 1.6), ISS III (RR: 1.7), and elevated LDH (RR: 1.3) were independently associated with decreased overall survival (OS). Among 1327 evaluable patients, OS was 11.0 (95\% Cl: 9.2-12.6), 7.0 (95\% Cl: 6.3-9.2), and 4.5 (95\% Cl: 3.7-5.2) years in patients with 0 (stage I), 1 (stage II), and $\geq 2$ (stage III) high-risk factors, respectively. In the MMRF cohort, median OS was 7.8 (95\% Cl: NR-NR), 6.0 (95\% Cl: 5.7-NR), and 4.3 (95\% Cl: 2.7-NR) years in the 3 groups, respectively $(P<0.001)$. This 5 -factor, 3-tier system is easy to implement in practice and improves upon the current R-ISS.

Blood Cancer Journal (2022)12:21; https://doi.org/10.1038/s41408-022-00611-x

\section{INTRODUCTION}

Multiple myeloma (MM) is the second most common hematologic malignancy and is responsible for approximately $2 \%$ of all cancer deaths in the United States [1]. With the advent of novel therapeutic agents and drug combinations, survival outcomes have improved considerably [2]. Despite these therapeutic advances, survival outcomes remain highly variable even in uniformly treated clinical trial populations [3, 4]. This disparity highlights the importance of risk stratification at the time of diagnosis for prognostication and patient selection for clinical trials $[5,6]$. Several risk stratification systems have been proposed based on clinical characteristics, laboratory studies, bone marrow cytogenetics, and gene expression profiling. Among them, the International Staging System (ISS) and its successor, the revised ISS (R-ISS), have stood the test of time in clinical practice. The ISS was first introduced in 2005 and is based on $\beta 2$-microglobulin and albumin, which are thought to reflect tumor burden and host status [7]. In clinical practice, the ISS remains a popular choice for risk stratification due to its simplicity, although its ability to discriminate among lower-risk patients is limited in the era of novel therapies [8]. Interphase fluorescence in-situ hybridization (FISH) enabled the identification of high-risk disease independent of ISS stage based on abnormalities such as $\mathrm{t}(4 ; 14), \mathrm{t}(14 ; 16)$, and $\operatorname{del}(17 \mathrm{p})[9,10]$. The revised ISS (R-ISS) was introduced in 2015 and includes these cytogenetic abnormalities as well as elevated lactate dehydrogenase (LDH) as high-risk markers [11]. The R-ISS has subsequently been validated in several studies $[12,13]$ and is currently used to risk-stratify patients with newly diagnosed MM [11]. It is thought to perform better than its predecessor in certain patient populations $[8,12,13]$. Recent studies have identified additional cytogenetic abnormalities not included in the R-ISS to be associated with adverse survival outcomes including monosomy 13 [14, 15], gain/amplification of $1 \mathrm{q} 21[16,17]$, and rearrangements involving the MYC gene [18-20]. Even though more powerful high-risk classifiers have been developed based on gene expression profiles and cytogenetics, their acceptance in clinical practice remains poor due to the involved resources and need for complex modeling [21-25]. While the presence of high-risk laboratory and cytogenetic features has been incorporated into the R-ISS, the additive effects of multiple cooccurring high-risk disease features have not been accounted for $[15,16]$. Importantly, as RISS was developed, assessment of 1q abnormalities was not commonly employed and there was limited data to explore the value of adding this variable. Furthermore, unlike the ISS which had a similar proportion of patients in each of the three stages, R-ISS resulted in majority of patients being classified as intermediate. In light of the identification of additional cytogenetic risk factors and an improved understanding of the prognostic implications of multi-hit disease, we conducted this study to evaluate a new simple additive staging system in patients with newly diagnosed MM $[14,17,19,20]$.

${ }^{1}$ Division of Hematology, Mayo Clinic, Rochester, MN, USA. ${ }^{2}$ Department of Laboratory Medicine and Pathology, Rochester, MN, USA. ${ }^{3}$ Division of Nephrology and Hypertension, Mayo Clinic, Rochester, MN, USA. ${ }^{4}$ Division of Hematology, Mayo Clinic, Phoenix, AZ, USA. ${ }^{{ }_{\text {email: }}}$ kumar.shaji@mayo.edu

Received: 12 August 2021 Revised: 7 December 2021 Accepted: 12 January 2022

Published online: 31 January 2022 


\section{PATIENTS AND METHODS}

\section{Patients and study design}

We included all patients with MM seen in Mayo Clinic in Rochester, Minnesota between 2004 and 2019, who had cytogenetic analysis by FISH performed within 1 year before diagnosis, or within 6 months from the start of first-line treatment. The cohort included 2556 patients 18 years and older, diagnosed with MM between February 2004 and June 2019. Patients were identified from a preexisting database and additional data was obtained by review of electronic medical records. We extracted data on FISH results, standard serum and urine tests, bone marrow biopsy results, first-line treatment, transplant status, and date of death or last follow up. For model development, we included 1327 Mayo Clinic patients who had simultaneous data for all high-risk abnormalities found to be prognostic on multivariate analysis. The validation population included 502 patients enrolled in the MMRF CoMMpass study (MMRF); all data were obtained through the MMRF Researcher Gateway (https://research.themmrf.org). The study was approved by the Mayo Clinic Institutional Review Board. Informed consent was obtained from all patients included in the study.

\section{Cytogenetics}

In the Mayo population, FISH was performed on unsorted bone marrow plasma cells identified by cytoplasmic immunoglobulin staining, as previously described [26]. Only cytoplasmic immunoglobulin positive plasma cells were scored with the goal to reach 50 plasma cells per FISH probe set. The FISH panel included the following probes with their corresponding normal cutoffs (\%): 3 centromere (D3Z1, Abbott Molecular) (20\%), 7 centromere (D7Z1, Abbott Molecular) (20\%), 9 centromere (D9Z1, Abbott Molecular) (20\%), 15 centromere (D15Z4, Abbott Molecular) (20\%), -13q14 (RB1/LAMP1, Abbott Molecular) (20\%), -13q (RB1/LAMP1, Abbott Molecular) (20\%), -17p13.1 (TP53/D17Z1, Abbott Molecular) (20\%), and -17 (TP53/D17Z1, Abbott Molecular) (20\%), enumeration probes.

Dual-color, dual-fusion probes targeting $\mathrm{t}(11 ; 14) \mathrm{CCND} 1 / \mathrm{lgH}$ (Abbott Molecular) (6\%), and break apart probe targeting IgH (in-house developed) $(10 \%)$ were used. If an $\mathrm{IgH}$ rearrangement other than $\mathrm{t}(11 ; 14)$ was found by the IgH break apart probe, reflex testing was done using dual-color, dualfusion probes to identify the translocation partner: $\mathrm{t}(4 ; 14)(\mathrm{p} 16.3 ; \mathrm{q} 32)$ FGFR3/lgH (6\%), $\mathrm{t}(14 ; 16)(\mathrm{q} 32 ; \mathrm{q} 23) \mathrm{IgH} / \mathrm{MAF}(6 \%), \mathrm{t}(14 ; 20)(\mathrm{q} 32 ; \mathrm{q} 12) \mathrm{lgH} /$ MAFB (6\%), and $\mathrm{t}(6 ; 14)(\mathrm{p} 21 ; \mathrm{q} 32)$ CCND3/lgH (Abbott Molecular) $(6 \%)$. The $\mathrm{t}$ $(4 ; 14), t(14 ; 16)$, and $t(14 ; 20)$ translocations were considered high-risk [5]. Double- and triple hit disease were defined as the presence of a primary high-risk cytogenetic abnormality $[\mathrm{t}(4 ; 14), \mathrm{t}(14 ; 16), \mathrm{t}(14 ; 20)]$ with 1 or 2 additional high-risk cytogenetic abnormalities (chromosome 17 abnormality) and/or 1q gain/amplification), respectively [15]. The MYC break apart probe and $1 q / 1 p$ enumeration probes were introduced for clinical use as part of the Myeloma FISH panel in August 2014. For samples obtained before this date, testing for these cytogenetic abnormalities was performed as an add-on test using samples not subjected to cytoplasmic immunoglobulin counterstain. After this date, testing was performed on plasma cell-enriched samples using the cytoplasmic immunoglobulin stain. The methods used for $1 \mathrm{q}$ gain and $M Y C$ rearrangement testing were previously described $[17,20]$. 1q gain was determined using the ratio of $1 q 22$ to $1 p$ (TP73) using an in-house custom developed probe $(1 q / 1 p$ (1q22/TP73) (3.5\% for unselected plasma cell samples, 20\% for counterstained samples). A break apart probe targeting 8q24.1 (MYC, Abbott Molecular) was used to detect a MYC rearrangement $(6.5 \%$ for unselected plasma cell samples, $10 \%$ for counterstained samples). In the validation population, next generation sequencing based FISH (Seq-FISH) was used. This method has been validated and demonstrated comparable specificity and improved sensitivity compared to interphase FISH [27, 28]. Seq-FISH probes were readily available for IGH translocations, MYC rearrangement, 1q gain (20\% threshold), and chromosome 17 abnormalities.

\section{Statistical analysis}

Using the entire Mayo cohort $(n=2556)$, we first examined the impact of each of the cytogenetic abnormalities detected by FISH on overall survival (OS) using univariate analysis. These abnormalities included: (1) high-risk translocations involving the immunoglobulin heavy chain (lgH) locus, (2) t $(11 ; 14)$ translocation, (3) trisomies of at least 1 chromosome, (4) rearrangements involving the MYC gene locus, (5) gain of 1 or more copies of $1 \mathrm{q}(1 \mathrm{q}$ gain/amplification), (6) monosomy of chromosome 13 (not including patients with 13q deletion alone), and (7) chromosome 17 abnormalities [del(17p) or monosomy of chromosome 17]. Cytogenetic abnormalities significantly associated with survival in the univariate model were included in a multivariate model; those significantly associated with survival in the multivariate model were then included in a final multivariate model with ISS stage III (vs I/II) and elevated LDH. Values above the upper limit of normal of the reporting laboratory were considered elevated for lactate dehydrogenase (above $222 \mathrm{U} / \mathrm{l}$ for the Mayo Clinic Laboratories). ISS stages were defined as: $\beta 2$-microglobulin $<3.5 \mathrm{mg} / \mathrm{dL}$ and albumin $\geq 3.5 \mathrm{~g} / \mathrm{dL}$ (stage l); $\beta 2$ microglobulin $\geq 5.5$ regardless of albumin (stage III); all other cases were considered stage II [7]. For R-ISS, stage I included patients with ISS stage I, normal LDH, and absence of high-risk cytogenetics; R-ISS stage III included patients with ISS stage III, elevated LDH and/or high-risk cytogenetics; all other cases were considered stage II [11]. Overall survival (OS) was defined as the time from diagnosis of multiple myeloma to death from any cause. Progression-free survival (PFS) was defined as the time from the start of first-line treatment to first disease progression or death from any cause (whichever occurred first). Patients without an event at the end of follow-up were censored. Risk ratios (RR) were calculated based on the univariate and multivariate cox proportional hazards models.

\section{Model development and validation-The Mayo Additive Staging System (MASS)}

First, we updated the R-ISS such that the definition of HR cytogenetic abnormalities included all the FISH abnormalities found to be significantly associated with survival in our final multivariate model. We then compared the PFS and OS of patients based on the updated R-ISS. Next, given that the R-ISS does not give similar weight to all the prognostic factors, we explored a simpler approach that incorporates all the independent prognostic factors, and grouped patients by virtue of the number of risk factors. For this analysis, we included 1327 Mayo Clinic patients who had simultaneous data available for all the variables significantly associated with survival in the final multivariate model. We examined PFS and OS of patients based on the number of high-risk abnormalities; patients with 0,1 , or $\geq 2 \mathrm{HR}$ abnormalities were considered as stage I, II, or III, respectively. We then performed a subgroup analysis for OS based on age group $(<65$ years vs. $\geq 65$ years), transplant status, and across 2 time periods (before and after 2012). Next, we evaluated the performance of this model in an independent population of 502 patients enrolled in the MMRF CoMMpass study (MMRF). The performance of the model was measured by Harrell's Concordance Index (C) $[29,30]$. Overall and progression-free survival estimates were calculated using the Kaplan-Meier method, and survival was compared between groups using the Log-rank test [31]. For all tests, 2 -sided $P$ values $<0.05$ were considered statistically significant. Statistical analyses were performed using the JMP (SAS, Cary, NC) and Stata statistical softwares.

\section{RESULTS}

\section{Baseline patient characteristics}

The Mayo clinic cohort included 2556 patients diagnosed with MM between February 2004 and June 2019. The median age was 64 years and $62 \%$ were males. The clinical characteristics of patients and the first-line treatments are included in Table 1. Among all patients, $143(6 \%)$ had no abnormality detected by FISH using the probes tested. A trisomy of at least one chromosome was found in $55 \%$ of patients tested. $\mathrm{t}(11 ; 14)$ was the most common $\mathrm{IgH}$ translocation, found in $21 \% ; t(4 ; 14)$ and $t(14 ; 16)$ were found in $10 \%$ and $4 \%$, respectively. A gain of 1 or more copies of chromosome $1 \mathrm{q}$ was detected in $31 \%$, and a rearrangement involving the MYC gene locus was found in $9 \%$. A monosomy of chromosome 13 was seen in $37 \%$, and an abnormality in chromosome 17 [del(17p)/monosomy 17] was seen in 13\%. These results are presented in Table 2 .

\section{Univariate and multivariate survival analysis}

The median follow-up in the entire cohort was 6.2 (95\% Cl: 5.9-6.5) years. At the time of analysis, $58 \%$ of patients were alive; the median OS was 7.5 (95\% Cl: 7.0-8.1) years. On univariate analysis, high-risk IgH translocations (RR: 2.0), MYC rearrangements (RR: 1.5), 1q gain/amplification (RR: 1.8), monosomy of chromosome 13 (RR: 1.4), and chromosome 17 abnormalities (RR: 2.0), were all associated with increased risk of death, while the presence of trisomies was associated with decreased risk of death (RR: 0.8); $t$ $(11 ; 14)$ was not prognostic for OS (RR: 1.0). On multivariate analysis 
Table 1. Baseline characteristics.

\begin{tabular}{|c|c|c|}
\hline Baseline characteristics & $\begin{array}{l}\text { Median } \\
\text { (interquartile range) }\end{array}$ & $N(\%)$ \\
\hline Age (years) & $64(57-71)$ & \\
\hline Male & & $1582(62)$ \\
\hline ECOG PS $\geq 2$ (vs $0-1)$ & & $141(20)$ \\
\hline Hemoglobin (g/dL) & $11.0(9.5-12.5)$ & \\
\hline Hemoglobin $\leq 10 \mathrm{~g} / \mathrm{dL}$ & & $720(33)$ \\
\hline Platelets $\left(\times 10^{9} / \mathrm{L}\right)$ & $210(162-262)$ & \\
\hline Creatinine (mg/dL) & $1.1(0.9-1.5)$ & \\
\hline Creatinine $\geq 2 \mathrm{mg} / \mathrm{dL}$ & & $319(16)$ \\
\hline LDH > ULN (units/L) & & $298(17)$ \\
\hline B2M ( $\mu \mathrm{g} / \mathrm{ml})$ & $4.0(2.7-6.6)$ & \\
\hline B2M > 5.5 vs. $(\leq 5.5)$ & & $701(32)$ \\
\hline Albumin (g/dL) & $3.6(3.2-3.9)$ & \\
\hline Albumin $\leq 3.5$ (vs. $>3.5$ ) & & $928(48)$ \\
\hline Calcium (mg/dL) & $9.5(9.0-10.1)$ & \\
\hline Calcium $\geq 11 \mathrm{mg} / \mathrm{dL}$ & & $215(11)$ \\
\hline Serum M spike (g/dL) & $2.5(0.7-3.9)$ & \\
\hline Urine $M$ spike (g/24 h) & $0.05(0-0.50)$ & \\
\hline Urine albumin $(g / 24 h)$ & $0.05(0.02-0.14)$ & \\
\hline $\operatorname{IgA} M M$ & & $464(25)$ \\
\hline IgG MM & & $1177(62)$ \\
\hline LC MM & & $214(11)$ \\
\hline \multicolumn{3}{|l|}{ Involved LC } \\
\hline Kappa & & $1252(65)$ \\
\hline Lambda & & $674(35)$ \\
\hline ISS Stage III (vs. I\&II) & & $710(33)$ \\
\hline BMPCs (\%) & $50(30-70)$ & \\
\hline PCLI (\%) & $0.8(0.3-1.5)$ & \\
\hline \multicolumn{3}{|l|}{ First-line treatment } \\
\hline PI & & $727(31)$ \\
\hline IMiD & & $720(31)$ \\
\hline $\mathrm{PI}+\mathrm{IMiD}$ & & $804(34)$ \\
\hline Other & & $107(5)$ \\
\hline Transplant & & $1399(55)$ \\
\hline $\begin{array}{l}\text { Early ( } \leq 1 \text { year from } \\
\text { diagnosis) }\end{array}$ & & $1184(85)$ \\
\hline $\begin{array}{l}\text { Late (>1 year from } \\
\text { diagnosis) }\end{array}$ & & $215(15)$ \\
\hline
\end{tabular}

Clinical and laboratory characteristics at diagnosis of patients diagnosed with multiple myeloma included in the study. The median (range) are presented for continuous variables and number (percentage) for categorical variables.

$B 2 M$ beta2microglobulin, $B M P C s$ bone marrow plasma cells, IMiD immunomodulatory drug, ISS international staging system, $L C$ light chain, $L D H$ lactate dehydrogenase, $M M$ multiple myeloma, $P C L$ plasma cell labeling index, PI proteasome inhibitor, PS performance status, ULN upper limit of normal.

including all FISH abnormalities significantly associated with OS on univariate analysis, monosomy 13 (RR: $1.1, P=0.20$ ) and trisomies (RR: $0.9, P=0.08$ ) were no longer prognostic for OS. ISS III and elevated LDH were both associated with increased risk of death on univariate analysis, so these were included in a final multivariate model with high-risk lgH translocations, MYC rearrangements, 1q gain/amplification, and chromosome 17 abnormalities. HR $\operatorname{lgH}$ translocations (RR: 1.7, $P<0.001$ ), 1q gain/amplification (RR: 1.4,
Table 2. Cytogenetic abnormalities in multiple myeloma patients.

\begin{tabular}{lcc}
$\begin{array}{l}\text { Primary abnormalities } \\
\text { IgH translocations }\end{array}$ & Tested $\boldsymbol{N}$ & Abnormality $\boldsymbol{N}(\%)$ \\
\hline $\mathrm{t}(4 ; 14)$ & 2519 & $248(10)$ \\
\hline $\mathrm{t}(14 ; 16)$ & 2517 & $99(4)$ \\
\hline $\mathrm{t}(11 ; 14)$ & 2522 & $519(21)$ \\
\hline Trisomies & 2491 & $1374(55)$ \\
\hline Secondary abnormalities & & \\
\hline 19 gain/amplification & 1896 & $585(31)$ \\
\hline $\begin{array}{l}\text { Chromosome 17 abnormality } \\
\text { (17pdel/monosomy 17) }\end{array}$ & 2499 & $337(13)$ \\
\hline Monosomy 13 & 2513 & $926(37)$ \\
\hline MYC rearrangement & 1856 & $160(9)$
\end{tabular}

Prevalence of recurrent primary and secondary cytogenetic abnormalities in patients tested by FISH at diagnosis.

$\mathrm{IgH}$ immunoglobulin heavy chain gene locus, del deletion.

$P<0.001)$, chromosome 17 abnormalities (RR: $1.6, P<0.001)$, ISS III (RR: 1.7, $P<0.001$ ), and elevated LDH (RR: $1.3, P=0.01$ ) were all independently associated with decreased OS in the final multivariate model. MYC rearrangements were associated with decreased survival, but this was not statistically significant (RR: $1.3, P=0.06)$. These results are presented in Table 3 .

\section{PFS and OS based on the updated R-ISS}

The R-ISS was updated to include $1 \mathrm{q}$ gain/amplification in the definition of high-risk cytogenetic abnormalities, in addition to high-risk lgH translocations and chromosome 17 abnormalities. Based on this definition, 193 (11\%), 1130 (66\%), and 396 (23\%) patients had stage I, II, and III disease, respectively. The median PFS was 60.0 (95\% Cl: 46.1-87.1), 44.0 (95\% Cl: 40.5-48.8), and 28.1 (95\% Cl: $23.0-31.4)$ months in the 3 groups, respectively $(P<0.001)$ (Fig. 1a). Median OS was 9.4 (95\% Cl: 8.9-12.8), 7.5 (95\% Cl: 6.4-8.0), and 3.9 (95\% Cl: 3.6-4.6) years in patients with stage I, II, and III disease, respectively $(P<0.001)$ (Fig. 1b).

\section{Developing a simpler approach for risk stratification - The MASS}

Among all patients included in the study, 1327 had simultaneous data available for: high-risk IgH translocations, 1q gain/amplification, chromosome 17 abnormalities, ISS stage, and LDH. There were no significant differences in baseline characteristics between evaluable patients and the rest of the cohort (Supplemental Table 1). Among evaluable patients, 476 (36\%) had no high-risk factors (stage I), 442 (33\%) had 1 high-risk factor (stage II), and 409 (31\%) had $\geq 2$ high-risk factors (stage III). Median PFS was 63.1 (95\% Cl: $53.0-70.8), 44.0$ (95\% Cl: $37.8-58.7)$, and 28.6 (95\% Cl: 25.4-34.7) months in the 3 groups, respectively (Fig. 2a). OS was 11.0 (95\% Cl: 9.2-12.6), 7.0 (95\% Cl: 6.3-9.2), and 4.5 (95\% Cl: 3.7-5.2) years in patients with stage I, II, and III disease, respectively (Fig. 2b) (Table 4).

\section{Subgroup analysis by age and transplant status}

The prognostic ability of this staging system was evaluated based on age group and transplant status. Among patients $<65$ years, the median OS was 12.8 (95\% Cl: 11.3-NR), 9.3 (95\% Cl: 6.6-11.5), and 5.5 (95\% Cl: 3.9-7.0) years in patients with stage I, II, and III disease, respectively $(P<0.001)$ (Fig. 3a). In patients $\geq 65$ years, the median OS was 8.3 (95\% Cl: 6.3-9.1), 6.4 (95\% Cl: 5.2-7.7), and $3.7(95 \% \mathrm{Cl}$ : 3.2-4.5) years in the 3 groups, respectively $(P<0.001)$ (Fig. 3b). Among patients who did not undergo transplant, median OS was 9.1 (95\% Cl: 6.5-11.5), 5.8 (95\% Cl: 5.0-6.7), and 3.0 (95\% Cl: 2.3-3.4) years in patients with stage I, II, and III disease, respectively $(P<$ 
Table 3. Univariate and multivariate survival models.

\begin{tabular}{|c|c|c|c|c|c|c|}
\hline \multirow[t]{2}{*}{ Variable } & \multicolumn{2}{|l|}{ Univariate } & \multicolumn{2}{|c|}{$\begin{array}{l}\text { Multivariate (FISH } \\
\text { abnormalities only) }\end{array}$} & \multicolumn{2}{|l|}{ Multivariate (all) } \\
\hline & OS RR $(95 \% \mathrm{Cl})$ & $P$ value & OS RR $(95 \% \mathrm{CI})$ & $P$ value & OS RR $(95 \% \mathrm{Cl})$ & $P$ value \\
\hline HR IgH translocations & $2.0(1.7-2.3)$ & $<0.001$ & $1.6(1.3-1.9)$ & $<0.001$ & $1.7(1.3-2.1)$ & $<0.001$ \\
\hline$t(11 ; 14)$ & $1.0(0.8-1.2)$ & 0.88 & - & - & - & - \\
\hline Trisomies & $0.8(0.7-0.9)$ & 0.003 & $0.9(0.7-1.0)$ & 0.08 & - & - \\
\hline MYC rearrangement & $1.5(1.2-1.9)$ & $<0.001$ & $1.5(1.2-1.9)$ & 0.002 & $1.3(1.0-1.8)$ & 0.06 \\
\hline Monosomy 13 & $1.4(1.2-1.6)$ & $<0.001$ & $1.1(0.9-1.3)$ & 0.20 & - & - \\
\hline 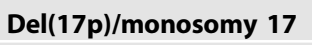 & $2.0(1.7-2.3)$ & $<0.001$ & $1.9(1.6-2.3)$ & $<0.001$ & $1.6(1.3-2.0)$ & $<0.001$ \\
\hline ISS III (vs. ISS I/II) & $1.9(1.7-2.2)$ & $<0.001$ & - & - & $1.7(1.4-2.0)$ & $<0.001$ \\
\hline Elevated LDH & $1.6(1.4-1.9)$ & 0.001 & - & - & $1.3(1.1-1.7)$ & 0.01 \\
\hline
\end{tabular}

Univariate and multivariate analysis including cytogenetic abnormalities, ISS stage and LDH.

Del deletion, $H R$ high-risk, IgH immunoglobulin heavy chain gene locus, ISS international staging system, LDH lactate dehydrogenase, OS overall survival, RR risk ratio. Bolded numbers represent $P$ values $<0.05$.
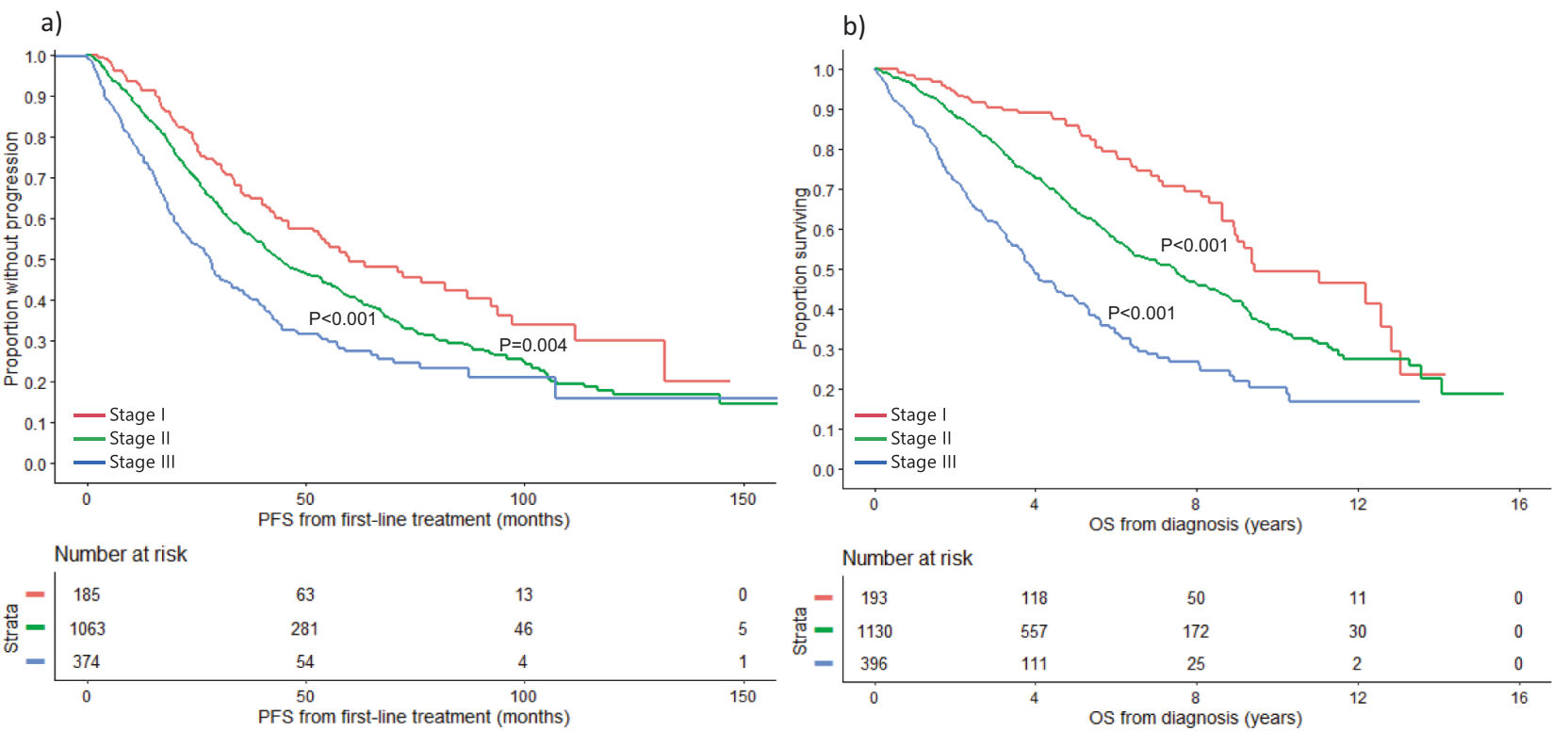

Fig. 1 PFS and OS based on the updated R-ISS. a PFS (months) and b OS (years) in MM patients with stage I (red curve), II (green), and III (blue curve) based on the updated R-ISS. MM multiple myeloma, OS overall survival, PFS progression-free survival, $R$-ISS revised international staging system. The $P$ values for each pair of groups are presented between the corresponding curves.

0.001) (Fig. 4a). In patients who underwent transplant, median OS was 11.3 (95\% Cl: 10.4-13.0), 9.7 (95\% Cl: $7.5-10.4)$, and $6.1(95 \% \mathrm{Cl}$ : $5.6-8.8)$ years in the 3 groups, respectively $(P<0.001)$ (Fig. 4b).

Risk stratification based on the MASS over 2 time periods Of all the patients included in the study, 1001 were diagnosed with MM prior to the year 2012, including 407 who had available data for staging by the MASS. Among those, 174 (43\%), 128 (31\%), and $105(26 \%)$ patients had stage I, II, and III disease by MASS, respectively. The median PFS was 36.7 (95\% Cl: $32.1-46.0), 31.3$ (95\% Cl: 26.6-36.4), 18.8 (95\% Cl: 13.3-21.8) months in the 3 groups, respectively $(P<0.001)$. The $O S$ in the 3 groups was 10.4 (95\% Cl: 8.8-11.5), 6.6 (95\% Cl: 5.5-8.7), and 3.3 (95\% Cl: 2.3-4.2) years, respectively $(P<0.001)$. Of the 1555 patients diagnosed with MM after year 2012, 920 had available data for MASS staging. Among those, 302 (33\%), 314 (34\%), and 304 (33\%) patients had stage I, II, and III disease by MASS, respectively. The median PFS was NR (95\% Cl: 74.5-NR), 75.6 (67.4-NR), and $43.4(95 \% \mathrm{Cl}$ :
37.4-57.5) months in the 3 groups, respectively $(P<0.001)$. The OS was NR (95\% Cl: NR-7.7-NR), NR (95\% Cl: 6.3-NR), and 5.7 (95\% Cl: 4.0-6.1) years, in the 3 groups, respectively, $(P<0.001)$ These results are shown in Supplemental Fig. 1.

On multivariate analysis including the MASS stage, age ( $\geq 65$ vs. $<65$ ), transplant status, and era of diagnosis (prior to vs. after 2012), the MASS stage retained its prognostic ability with statistically different survival differences seen between the stages; the risk ratio (RR) for death was 1.7 for stage II vs. I, 2.0 for stage III vs. II, and 3.3 for stage III vs. I ( $P<0.001$ between all pairs).

\section{Stage migration using the MASS}

Among all patients, 1269 patients had simultaneous data available for R-ISS stage and MASS, including 244 (18\%) patients with R-ISS I, 791 (62\%) with R-ISS II and 234 (18\%) with R-ISS III. Overall, 469 (37\%) had stage migration when the MASS was used for risk stratification (Fig. 5). Among R-ISS I patients, 21\% were reclassified as stage II using the MASS system; $32 \%$ and $21 \%$ of patients with 
a)

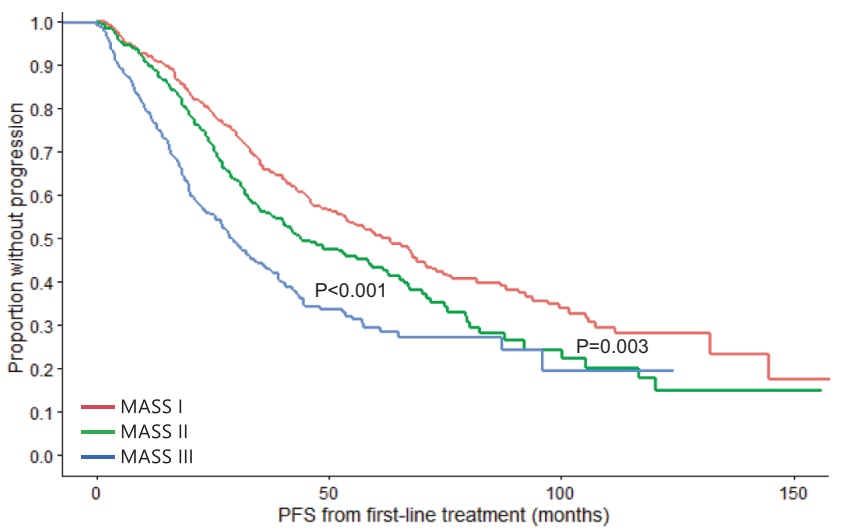

\begin{tabular}{|cccc} 
Number at risk & & \\
457 & 149 & 35 & 1 \\
416 & 94 & 12 & 2 \\
386 & 52 & 2 & 0 \\
\hline 0 & 50 & 100 \\
PFS from first-line treatment (months)
\end{tabular} b)

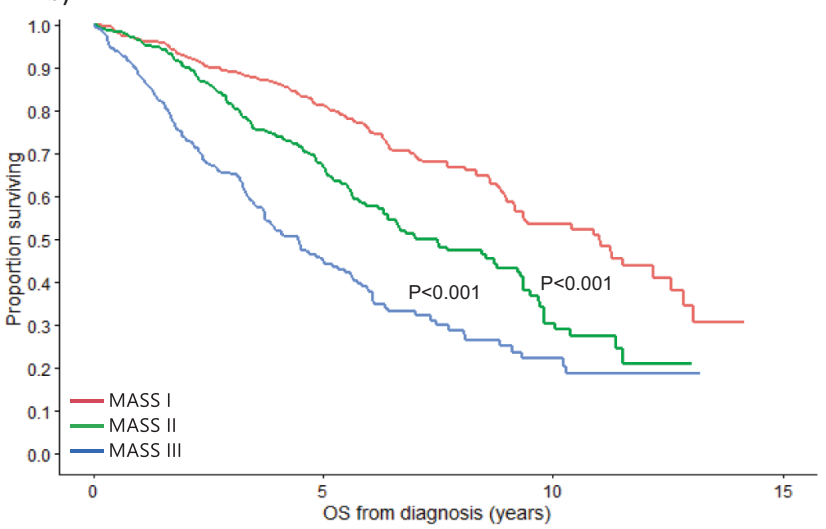

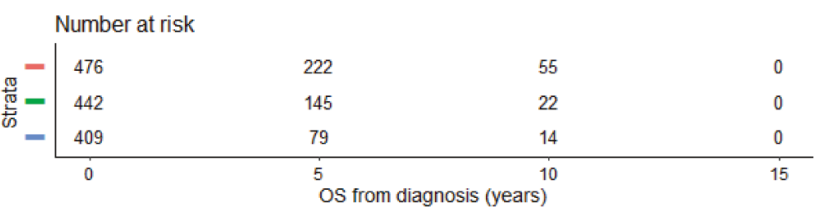

Fig. 2 PFS and OS based on the MASS using the Mayo Clinic cohort. a PFS (months) and b OS (years) in MM patients with no HR factors (stage I) (red curve), $1 \mathrm{HR}$ factor (stage II) (green curve), and $\geq 2$ HR factors (stage III) (blue curve). HR factors are defined as any of: HR IgH translocations, 1q gain/amplification, chromosome 17 abnormality [(del)17p/monosomy 17], ISS stage III, and LDH > ULN. del: deletion, HR: high-risk, IgH immunoglobulin heavy chain gene locus, ISS international staging system, LDH lactate dehydrogenase, MASS Mayo Additive Staging System, MM multiple myeloma, OS overall survival, PFS progression-free survival, ULN upper limit of normal. The $P$ values for each pair of groups are presented between the corresponding curves.

Table 4. MASS - Mayo Cohort.

\begin{tabular}{|c|c|c|c|c|c|}
\hline Predictor & Score & Total Score & Stage & PFS (months) & OS (years) \\
\hline High-risk IGH translocation & +1 & 0 & MASS I & 63.1 & 11.0 \\
\hline 1q gain/amplification & +1 & & & & \\
\hline Chromosome 17 abnormality & +1 & 1 & MASS II & 44.0 & 7.0 \\
\hline ISS stage III & +1 & & & & \\
\hline Elevated LDH & +1 & $2+$ & MASS III & 28.6 & 4.5 \\
\hline
\end{tabular}

The MASS and associated median progression-free and overall survival estimates for the MAYO population ( $n=1327$ ).

IGH immunoglobulin heavy chain locus, ISS International Staging System, LDH Lactate Dehydrogenase, MASS Mayo Additive Staging System, OS overall survival, PFS progression-free survival.

R-ISS II were classified as stages I and III, respectively using the MASS system. All patients in R-ISS III were classified as stage III using the MASS system. Among 147 patients with double-hit myeloma, 71 (48\%) were classified as stage II using the R-ISS and $76(52 \%)$ were classified as stage III. Among 17 patients with triple hit myeloma, 4 (24\%) were classified as R-ISS II and 13 (76\%) as R-ISS III. By definition, all patients with double and triple hit MM were classified as stage III using the MASS system. Stage migration from ISS to MASS is also shown in Fig. 5.

Among patients with R-ISS I, patients who were classified as MASS II had inferior OS (median OS: 6.8, 95\% Cl: 6.3-9.4 years) compared to patients classified as MASS I (median OS: $9.4,95 \% \mathrm{Cl}$ : 8.9-12.8 years), $P=0.03$ (supplemental Fig. 2a). Among patients with R-ISS II, median OS was 11.0 (95\% Cl: 8.9-NR), $7.0(95 \% \mathrm{Cl}$ : 5.9-9.3), and 4.8 (95\% Cl: 3.5-5.8) years in patients with MASS I, II, and III, respectively $(P<0.001)$ (supplemental Fig. 2b). Among patients with ISS I, those who were classified as MASS II (median OS: $8.7,95 \% \mathrm{Cl}$ : $6.3-9.8$ years) had decreased OS compared to patients with MASS I (median OS: 9.4, 95\% Cl: 8.9-12.8 years) $(P=$ 0.01 ), but no significant difference in OS compared to patients with MASS III (median OS: $7.7,95 \% \mathrm{Cl}$ : 3.3-NR years) $(P=0.39)$ (Supplemental Fig. 2C). Among patients with ISS II, a higher MASS stage was associated with inferior survival; median OS was 11.0 (95\% Cl: 8.9-NR), 7.5 (95\% Cl: 5.7-9.5), and 3.9 (95\% Cl: $3.2-5.7)$ years in patients with MASS I, II, and III, respectively $(P<0.001)$ (supplemental Fig. 2d).

\section{Validation using the MMRF cohort}

The MMRF cohort included 502 patients with a median age of 63 (IQR: $56-69)$ years; $57 \%$ were male. Median follow up was $4.4(95 \%$ Cl: 4.3-4.6) years. Among all 502 patients, 172 (34\%), 181 (36\%), and 149 (30\%) were classified as stage I, II, and III, respectively. Median PFS was 65.2 (95\% Cl: 47.2-NR), 38.0 (95\% Cl: 33.9-44.5), and 22.6 (95\% Cl: 18.1-26.3) months in patients with stage I, II, and III disease, respectively $(P<0.001)$ (Fig. 6a). Median OS was 7.8 (95\% Cl: NR-NR), 6.0 (95\% Cl: 5.7-NR), and 4.3 (95\% Cl: 2.7-NR) years in the 3 groups, respectively $(P<0.001)$ (Fig. 6b). The Harrell's $C$ for the MASS was $0.572(95 \% \mathrm{Cl} 0.547-0.598)$ compared to 0.560 (95\% Cl: 0.536-0.585) for the R-ISS.

\section{The MASS as a 4-tier staging system}

When the MASS was used as a 4-tier staging system in the Mayo cohort, OS was 11.0 (95\% Cl: 9.2-12.6), 7.0 (95\% Cl: 6.3-9.2), 5.0 (95\% Cl: 4.1-5.8), and 3.4 (95\% Cl: $2.6-4.1)$ years in patients with 

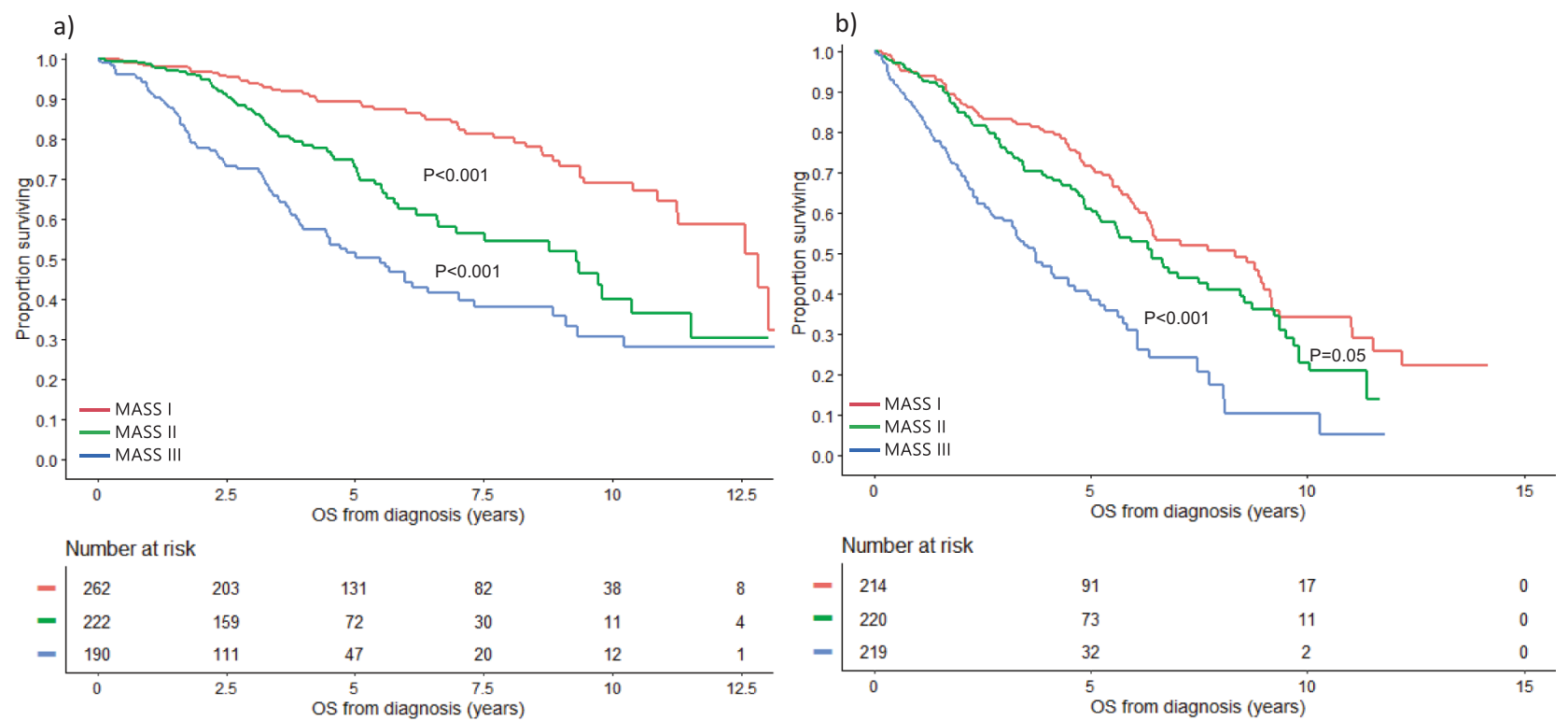

Fig. 3 OS based on the MASS by age. OS (years) in MM patients with MASS I (red curve), MASS II (green curve), and MASS III (blue curve) who are $\mathbf{a}<65$ and $\mathbf{b} \geq 65$ years of age. MASS Mayo Additive Staging System, MM multiple myeloma, OS overall survival. The $P$ values for each pair of groups are presented between the corresponding curves.

a)

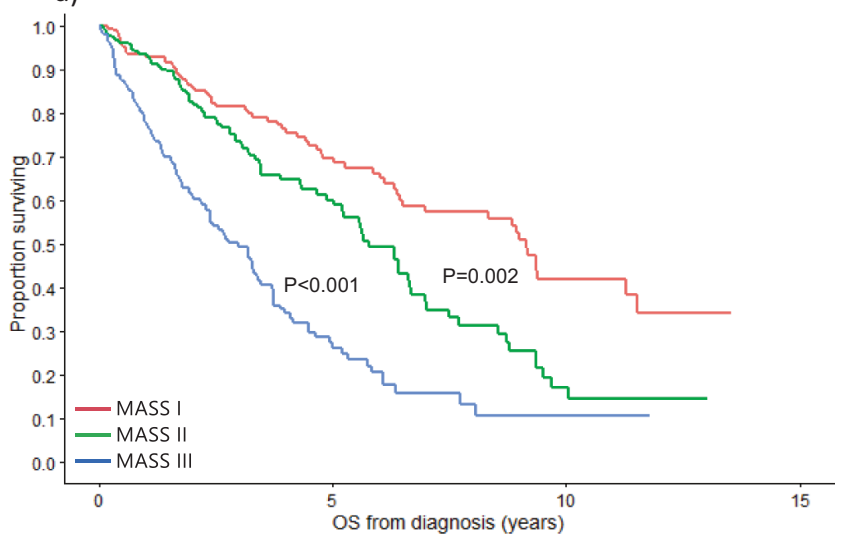

\begin{tabular}{llll} 
& & \\
& Number at risk \\
- & 68 & 18 & 0 \\
190 & 47 & 7 & 0 \\
189 & 20 & 3 & 0 \\
\hline 205 & 5 & 10 & 15 \\
\hline 0 & OS from diagnosis (years)
\end{tabular} b)

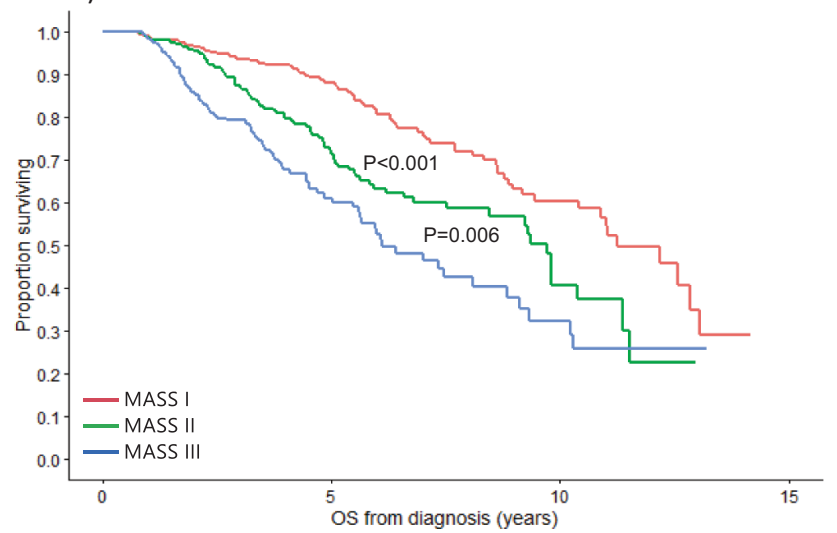

Number at risk

\begin{tabular}{llll} 
& Number at risk \\
$-\mid$ & & & \\
286 & 154 & 37 & 0 \\
253 & 98 & 15 & 0 \\
- & 59 & 11 & 0 \\
\hline 0 & 5 & 10 & 15
\end{tabular}

Fig. 4 OS based on the MASS by transplant status. OS (years) in MM patients with MASS I (red curve), MASS II (green curve), and MASS III (blue curve) who (a) did not undergo transplant and in those who (b) underwent transplant. MASS Mayo Additive Staging System, MM multiple myeloma, OS overall survival. The $P$ values for each pair of groups are presented between the corresponding curves.

stage I (no risk factors), stage II (1 risk factor), stage III (2 risk factors), and stage IV ( $\geq 3$ risk factors), respectively $(P<0.001)$. In the MMRF cohort, OS was 7.8 (95\% Cl: NR-NR), 6.0 (95\% Cl: 5.7-NR), 4.6 (95\% Cl: 3.7-5.9), and $1.6(95 \% \mathrm{Cl}: 1.1-3.7)$ years in patients with stage I, II, III, and IV disease, respectively $(P<0.001)$ (Supplemental Fig. 3$)$. The Harrell's C for the 4-tier MASS was 0.581 ( $95 \% \mathrm{Cl} 0.554-0.609)$.

\section{Impact of the number and type of HR cytogenetic} abnormalities

We evaluated the prognosis based on the number of $H R$ cytogenetic abnormalities (HR IgH translocation, 1q gain/amplification, chromosome 17 abnormality (del) $17 \mathrm{p} /$ monosomy 17 , and MYC rearrangement), excluding non-cytogenetic HR parameters of the MASS system (high LDH and ISS III). Median PFS was 57.9 (95\% Cl: 53.0-63.7), 40.2 (95\% Cl: 35.2-48.8), 31.1 (95\% Cl: 26.1-38.1), and 21.9 (95\% Cl: 15.7-42) months in patients with $0,1,2$, and $\geq 3$ HR cytogenetic abnormalities (Supplemental Fig. 4a). The corresponding median OS was $10.9(95 \% \mathrm{Cl}$ : 9.3-11.5), $5.6(95 \% \mathrm{Cl}$ : 5.2-6.3), 4.8 (95\% Cl: 3.7-6.1), and 3.1 (95\% Cl: 2.0-4.0) years, respectively (Supplemental Fig. 4b). Among patients with $1 \mathrm{HR}$ cytogenetic abnormality (610), median OS was 5.6, 5.7, 5.1, and 6.9 in patients with $\mathrm{HR} \mathrm{lgH}$ translocation, 1q gain/amplification, chromosome 17 abnormality, and MYC rearrangement, respectively; having a MYC rearrangement alone was associated with better survival compared to having a chromosome 17 abnormality (RR: $0.56, P=0.01$ ) or a HR IgH translocation (RR:0.70, $P=0.046$ ), 

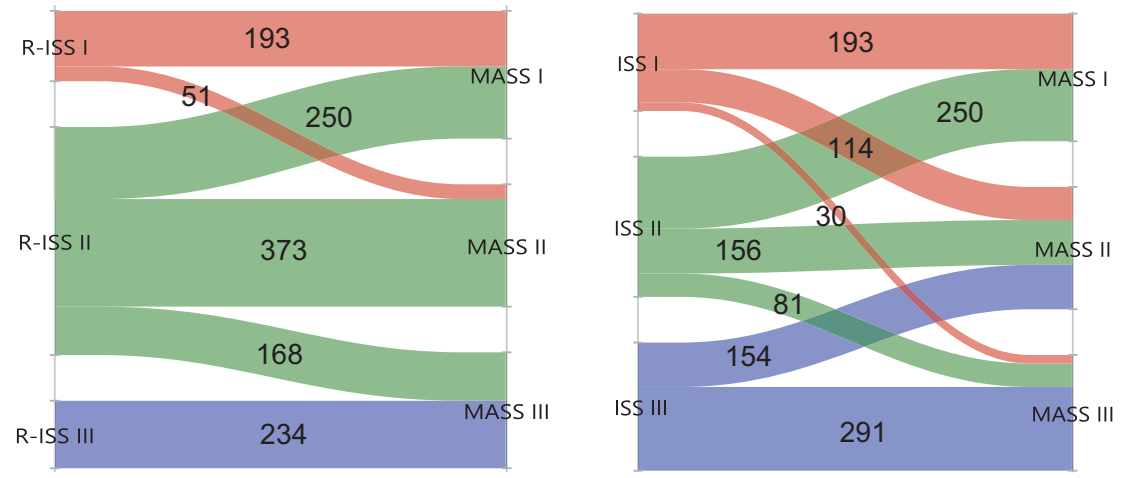

\begin{tabular}{|c||c||c|}
\hline $25 \%$ & $39 \%$ & $35 \%$ \\
\hline ISS I & ISS II & ISS III \\
\hline $17 \%$ & & \\
\hline R-ISS I & R-ISS II & R-ISS III \\
\hline $36 \%$ & $33 \%$ & $31 \%$ \\
\hline MASS I & MASS II & MASS III \\
\hline
\end{tabular}

Fig. 5 Stage migration between R-ISS and MASS and ISS and MASS. The distribution and migration of patients between disease stages using ISS, R-ISS, and MASS risk stratification systems. ISS International Staging System, R-ISS Revised International Staging System, MASS Mayo Additive Staging System.
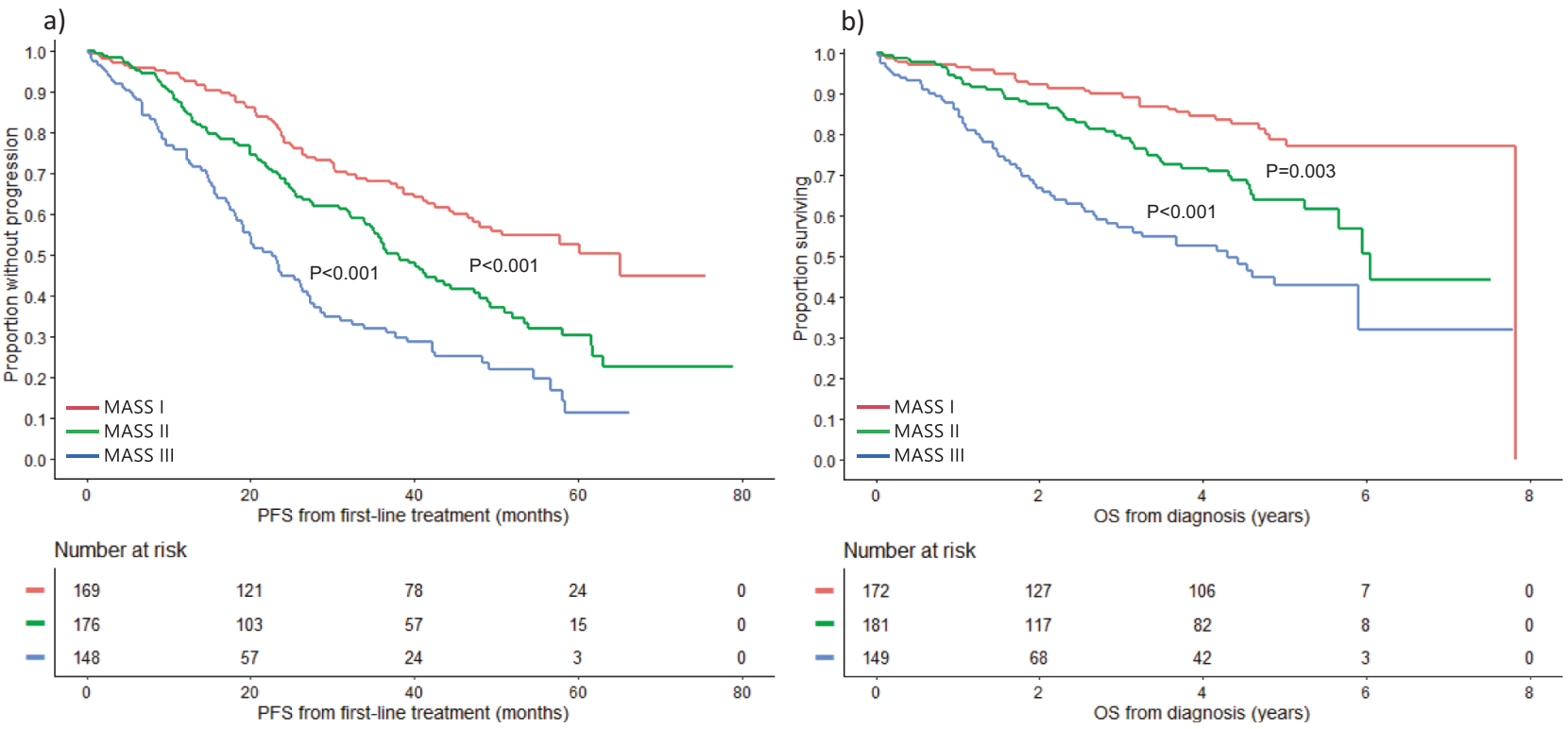

Fig. 6 PFS and OS based on the MASS using the MMRF cohort. a PFS (months) and b OS (years) in MM patients with MASS I (red curve), MASS II (green curve), and MASS III (blue curve) using the MMRF cohort. MASS Mayo Additive Staging System, MM multiple myeloma, OS overall survival, PFS progression-free survival. The $P$ values for each pair of groups are presented between the corresponding curves.

but there was no difference in OS between all other pairs. Among patients with two HR cytogenetic abnormalities (235 patients), there was no difference in OS between patients with one primary and one secondary HR abnormality, (median OS: $4.5,95 \% \mathrm{Cl}$ : 3.5-6.4 years) and those who had two secondary HR cytogenetic abnormalities $(5.7,95 \% \mathrm{Cl}: 2.9-8.1$ years $)(P=0.77)$. There was also no difference in OS associated with different combinations of HR cytogenetic abnormalities (global $P$ value: 0.66 ).

\section{DISCUSSION}

Risk stratification in MM remains an important concept both for patient counseling and the development of risk-adapted treatment strategies [5]. While minimal residual disease assessments are emerging as powerful predictors of survival outcomes for patients undergoing treatment, risk stratification in newly diagnosed patients has to rely on pre-treatment patient and disease characteristics [32]. The ISS and its successor, the R-ISS, 
have prevailed in clinical practice and research alike due to their simplicity and reliance on readily available baseline characteristics $[7,11]$. The recognition of additional risk factors motivated the revision of the ISS and resulted in a more powerful predictive model [11]. Since the introduction of the R-ISS, data on additional risk factors including chromosome 13 abnormalities, 1q gains, and MYC rearrangements have come to light [15-17, 20]. Furthermore, it has become evident that the co-occurrence of multiple high-risk disease features compounds the risk for adverse outcomes, leading to the concept of double- and triple-hit myeloma $[15,16]$. This insight led us to propose a refined additive risk stratification system.

We first evaluated the prognostic impact of known cytogenetic abnormalities in MM. In addition to the high-risk abnormalities included in the R-ISS, 1q gain demonstrated independent prognostic value in our final multivariate model, while the prognostic impact of MYC rearrangements did not reach statistical significance. Trisomies and monosomy 13 were not independently associated with OS when adjusting for other cytogenetic abnormalities, and thus the poor prognostic impact of nonhyperdiploidy [33] and chromosome 13 abnormalities [34-36] may be due to their association with other HR cytogenetic abnormalities. When we used an updated version of the R-ISS, where 1q gain/amplification was included in the definition of high-risk disease, we discriminated 3 groups of patients with significantly different outcomes. However, as with the original RISS, most patients had intermediate risk disease (stage II) using this classification (66\%). In addition, the R-ISS as originally designed did not consider the compounding effects of co-occurring highrisk disease features. Thus, we proposed a system that stratifies patients based on the number of high-risk factors present at diagnosis: HR IgH translocations, 1q gain/amplification, chromosome 17 abnormalities, ISS III, and/or elevated LDH. Using this system, we discriminated 3 groups of patients with significantly different progression-free and overall survival with nearly a third of the patients distributed between the 3 stages.

The prognostic utility of this system was demonstrated in both age groups: $\geq 65$ years and $<65$ years, in transplant-eligible and ineligible patients, and over 2 time periods. In addition, its prognostic value was shown in 2 independent populations. Compared to the R-ISS, the 3-tier MASS re-classified approximately one third of patients, underscoring how much our understanding of high-risk disease has been reshaped by the concept of multi-hit disease. Furthermore, the MASS retained its performance and discriminatory ability when used as a 4-tier risk stratification system which can be utilized if additional discrimination among high-risk patients is desired. This application has the potential to serve as an important tool in the design of clinical trials exploring intensification of treatments in high-risk patients. Importantly, this model will lend itself for incorporation of other risk factors as they are identified in the future.

Some limitations of this study include the long period of time over which data were collected during which treatments and transplant-eligibility criteria changed, and the exclusion of nonevaluable patients which may create selection bias. In addition, we did not evaluate some cytogenetic abnormalities for which data were not available like deletion $1 \mathrm{p} 32$ which has an estimated prevalence of $7 \%$ in newly diagnosed patients and has been associated with worse outcomes [37]. Furthermore, the lack of prognostic value of MYC abnormality in this study may be due to the limited number of patients with available cytogenetic data for $M Y C$, and thus its added value in risk stratification requires further evaluation in larger studies. The impact of the clonal plasma cell percentage harboring secondary cytogenetic abnormalities and the genetic mutational profile on risk stratification were not assessed in this study and also warrant exploration in future studies.

\section{CONCLUSION}

In summary, we developed and validated a simple, additive 5-factor 3-tier risk model for newly diagnosed MM in two diverse patient populations that is easy to implement in clinical practice and can play an important role in patient selection for clinical trials.

\section{REFERENCES}

1. Siegel RL, Miller KD, Jemal A. Cancer statistics, 2020. CA Cancer J Clin. 2020;70:7-30.

2. Kumar SK, Dispenzieri A, Lacy MQ, Gertz MA, Buadi FK, Pandey S, et al. Continued improvement in survival in multiple myeloma: changes in early mortality and outcomes in older patients. Leukemia 2014;28:1122-8.

3. Durie BGM, Hoering A, Sexton R, Abidi MH, Epstein J, Rajkumar SV, et al. Longer term follow-up of the randomized phase III trial SWOG S0777: bortezomib, lenalidomide and dexamethasone vs. lenalidomide and dexamethasone in patients (Pts) with previously untreated multiple myeloma without an intent for immediate autologous stem cell transplant (ASCT). Blood Cancer J. 2020;10:53.

4. Srivastava G, Rana V, Lacy MQ, Buadi FK, Hayman SR, Dispenzieri A, et al. Longterm outcome with lenalidomide and dexamethasone therapy for newly diagnosed multiple myeloma. Leukemia 2013;27:2062-6.

5. Chng WJ, Dispenzieri A, Chim CS, Fonseca R, Goldschmidt H, Lentzsch S, et al. IMWG consensus on risk stratification in multiple myeloma. Leukemia 2014:28:269-77.

6. Kyle RA, Gertz MA, Witzig TE, Lust JA, Lacy MQ, Dispenzieri A, et al. Review of 1027 patients with newly diagnosed multiple myeloma. Mayo Clin Proc. 2003;78:21-33.

7. Greipp PR, San Miguel J, Durie BG, Crowley JJ, Barlogie B, Blade J, et al. International staging system for multiple myeloma. J Clin Oncol. 2005;23:3412-20.

8. Cho H, Yoon DH, Lee JB, Kim SY, Moon JH, Do YR, et al. Comprehensive evaluation of the revised international staging system in multiple myeloma patients treated with novel agents as a primary therapy. Am J Hematol. 2017;92:1280-6.

9. Fonseca R, Bergsagel PL, Drach J, Shaughnessy J, Gutierrez N, Stewart AK, et al. International Myeloma Working Group molecular classification of multiple myeloma: spotlight review. Leukemia 2009;23:2210-21.

10. Avet-Loiseau H, Durie BG, Cavo M, Attal M, Gutierrez N, Haessler J, et al. Combining fluorescent in situ hybridization data with ISS staging improves risk assessment in myeloma: an International Myeloma Working Group collaborative project. Leukemia 2013;27:711-7.

11. Palumbo A, Avet-Loiseau H, Oliva S, Lokhorst HM, Goldschmidt H, Rosinol L, et al. Revised international staging system for multiple myeloma: a report from international myeloma working group. J Clin Oncol. 2015;33:2863-9.

12. Kastritis E, Terpos E, Roussou M, Gavriatopoulou M, Migkou M, EleutherakisPapaiakovou E, et al. Evaluation of the Revised International Staging System in an independent cohort of unselected patients with multiple myeloma. Haematologica 2017;102:593-9.

13. Tandon N, Rajkumar SV, LaPlant B, Pettinger A, Lacy MQ, Dispenzieri A, et al. Clinical utility of the Revised International Staging System in unselected patients with newly diagnosed and relapsed multiple myeloma. Blood Cancer J. 2017;7: e528.

14. Fonseca R, Harrington D, Oken MM, Dewald GW, Bailey RJ, Van Wier SA, et al. Biological and prognostic significance of interphase fluorescence in situ hybridization detection of chromosome 13 abnormalities (delta13) in multiple myeloma: an eastern cooperative oncology group study. Cancer Res. 2002;62:715-20.

15. Binder M, Rajkumar SV, Ketterling RP, Greipp PT, Dispenzieri A, Lacy MQ, et al. Prognostic implications of abnormalities of chromosome 13 and the presence of multiple cytogenetic high-risk abnormalities in newly diagnosed multiple myeloma. Blood Cancer J. 2017;7:e600.

16. Shah V, Sherborne AL, Walker BA, Johnson DC, Boyle EM, Ellis $S$, et al. Prediction of outcome in newly diagnosed myeloma: a meta-analysis of the molecular profiles of 1905 trial patients. Leukemia 2018;32:102-10.

17. Abdallah N, Greipp P, Kapoor P, Gertz MA, Dispenzieri A, Baughn LB, et al. Clinical characteristics and treatment outcomes of newly diagnosed multiple myeloma with chromosome 1q abnormalities. Blood Adv. 2020;4:3509-19.

18. Glitza IC, Lu G, Shah R, Bashir Q, Shah N, Champlin RE, et al. Chromosome 8q24.1/ c-MYC abnormality: a marker for high-risk myeloma. Leuk Lymphoma. 2015;56:602-7.

19. Walker BA, Wardell CP, Brioli A, Boyle E, Kaiser MF, Begum DB, et al. Translocations at 8 q24 juxtapose MYC with genes that harbor superenhancers resulting in overexpression and poor prognosis in myeloma patients. Blood Cancer J. 2014;4: e191. 
20. Abdallah N, Baughn LB, Rajkumar SV, Kapoor P, Gertz MA, Dispenzieri A, et al. Implications of MYC rearrangements in newly diagnosed multiple myeloma. Clin Cancer Res. 2020;26:6581-8.

21. Kuiper R, Broyl A, de Knegt $Y$, van Vliet $M H$, van Beers $E H$, van der Holt $B$, et al. $A$ gene expression signature for high-risk multiple myeloma. Leukemia 2012;26:2406-13.

22. Kuiper $R$, van Duin $M$, van Vliet $M H$, Broijl $A$, van der Holt B, El Jarari $L$, et al. Prediction of high- and low-risk multiple myeloma based on gene expression and the international staging system. Blood. 2015;126:1996-2004.

23. Mason MJ, Schinke C, Eng CLP, Towfic F, Gruber F, Dervan A, et al. Multiple myeloma DREAM challenge reveals epigenetic regulator PHF19 as marker of aggressive disease. Leukemia 2020;34:1866-74.

24. Perrot A, Lauwers-Cances V, Tournay E, Hulin C, Chretien ML, Royer B, et al. Development and validation of a cytogenetic prognostic index predicting survival in multiple myeloma. J Clin Oncol. 2019;37:1657-65.

25. Shaughnessy JD Jr, Zhan F, Burington BE, Huang Y, Colla S, Hanamura I, et al. A validated gene expression model of high-risk multiple myeloma is defined by deregulated expression of genes mapping to chromosome 1. Blood 2007;109:2276-84.

26. Fonseca R, Blood E, Rue M, Harrington D, Oken MM, Kyle RA, et al. Clinical and biologic implications of recurrent genomic aberrations in myeloma. Blood 2003;101:4569-75.

27. Goldsmith SR, Fiala MA, Dukeman J, Ghobadi A, Stockerl-Goldstein K, Schroeder $M A$, et al. Next generation sequencing-based validation of the revised international staging system for multiple myeloma: an analysis of the MMRF CoMMpass study. Clin Lymphoma Myeloma Leuk. 2019;19:285-9.

28. Miller C, Yesil J, Derome M, Donnelly A, Marrian J, McBride K, et al. A comparison of clinical FISH and sequencing based FISH estimates in multiple myeloma: an Mmrf CoMMpass analysis. Blood 2016;128:374.

29. Harrell FE Jr, Lee KL, Califf RM, Pryor DB, Rosati RA. Regression modelling strategies for improved prognostic prediction. Stat Med. 1984;3:143-52.

30. Newson RB. Comparing the predictive powers of survival models using Harrell's $C$ or Somers' D. Stata J. 2010;10:339-58.

31. Kaplan EL, Meier P. Nonparametric estimation from incomplete observations. J Am Stat Assoc. 1958;53:457-81.

32. Munshi NC, Avet-Loiseau H, Anderson KC, Neri P, Paiva B, Samur M, et al. A large meta-analysis establishes the role of MRD negativity in long-term survival outcomes in patients with multiple myeloma. Blood Adv. 2020;4:5988-99.

33. Smadja NV, Bastard C, Brigaudeau C, Leroux D, Fruchart C, Groupe Francais de Cytogenetique $\mathrm{H}$. Hypodiploidy is a major prognostic factor in multiple myeloma. Blood 2001;98:2229-38.

34. Fonseca R, Oken MM, Greipp PR, Eastern Cooperative Oncology Group Myeloma $\mathrm{G}$. The $\mathrm{t}(4 ; 14)(\mathrm{p} 16.3 ; \mathrm{q} 32)$ is strongly associated with chromosome 13 abnormalities in both multiple myeloma and monoclonal gammopathy of undetermined significance. Blood 2001;98:1271-2.

35. Chiecchio L, Protheroe RK, Ibrahim AH, Cheung KL, Rudduck C, Dagrada GP, et al Deletion of chromosome 13 detected by conventional cytogenetics is a critical prognostic factor in myeloma. Leukemia 2006;20:1610-7.

36. Chng WJ, Santana-Davila R, Van Wier SA, Ahmann GJ, Jalal SM, Bergsagel PL, et al. Prognostic factors for hyperdiploid-myeloma: effects of chromosome 13 deletions and IgH translocations. Leukemia 2006;20:807-13.

37. Hebraud B, Leleu X, Lauwers-Cances V, Roussel M, Caillot D, Marit G, et al Deletion of the $1 \mathrm{p} 32$ region is a major independent prognostic factor in young patients with myeloma: the IFM experience on 1195 patients. Leukemia 2014;28:675-9.

\section{ACKNOWLEDGEMENTS}

The MMRF kindly provided the data from the CoMMpass study for the purpose of scientific research. These data were generated as part of the Multiple Myeloma
Research Foundation Personalized Medicine Initiatives (https://research.themmrf.org and www.themmrf.org).

\section{AUTHOR CONTRIBUTIONS}

NA and SKK designed the study, collected, and analyzed the data, and wrote the first draft of the manuscript. $M B$ contributed to data analysis, provided critical revision and final approval of the manuscript version for publication. SVR, PTG, PK, AD, MAG LBB, MQL, SRH, FKB, DD, RSG, YLH, ALF, MAH, YL, NL, TK, RW, MAS, RAK, PLB, RF, RPK provided critical revision and final approval of the manuscript version for publication.

\section{COMPETING INTERESTS}

PK received research funding from Takeda Pharmaceuticals, Celgene, and Amgen. AD received research funding from Celgene, Millennium Pharmaceuticals, Pfizer, and Janssen and received a travel grant from Pfizer. MAG served as a consultant for Millennium Pharmaceuticals and received honoraria from Celgene, Millennium Pharmaceuticals, Onyx Pharmaceuticals, Novartis, GlaxoSmithKline, Prothena, Ionis Pharmaceuticals, and Amgen. MQL received research funding from Celgene. NL serves on an advisory board for Takeda Pharmaceuticals. RF served as a consultant for Amgen, BMS, Celgene, Takeda, Bayer, Janssen, Novartis, Pharmacyclics, Sanofi, Karyopharm, Merck, Juno, Kite, Aduro, OncoTracker, Oncopeptides, GSK, and AbbVie, and is on the scientific Advisory Board for Adaptive Biotechnologies, Caris Life Sciences and OncoTracker. SKK served as a consultant for Celgene, Millennium Pharmaceuticals, Onyx Pharmaceuticals, Janssen, and Bristol-Myers Squibb and received research funding from Celgene, Millennium Pharmaceuticals, Novartis, Onyx Pharmaceuticals, AbbVie, Janssen, and Bristol-Myers Squibb. The remaining authors declare no competing financial interests.

\section{ADDITIONAL INFORMATION}

Supplementary information The online version contains supplementary material available at https://doi.org/10.1038/s41408-022-00611-x.

Correspondence and requests for materials should be addressed to Shaji K. Kumar.

Reprints and permission information is available at http://www.nature.com/ reprints

Publisher's note Springer Nature remains neutral with regard to jurisdictional claims in published maps and institutional affiliations.

\begin{abstract}
Open Access This article is licensed under a Creative Commons Attribution 4.0 International License, which permits use, sharing, adaptation, distribution and reproduction in any medium or format, as long as you give appropriate credit to the original author(s) and the source, provide a link to the Creative Commons license, and indicate if changes were made. The images or other third party material in this article are included in the article's Creative Commons license, unless indicated otherwise in a credit line to the material. If material is not included in the article's Creative Commons license and your intended use is not permitted by statutory regulation or exceeds the permitted use, you will need to obtain permission directly from the copyright holder. To view a copy of this license, visit http://creativecommons. org/licenses/by/4.0/.
\end{abstract}

(c) The Author(s) 2022 